\begin{tabular}{lcr}
\hline \multicolumn{2}{c}{ A N N A L E S } \\
UNIVERSITATIS M A R I A E C URIE-SKŁ ODOWSK A \\
LOL. XXXVI & SECTIOFF & $1-2018$ \\
\hline
\end{tabular}

\title{
DOROTA LAJUS
}

ORCID ID: https://orcid.org/0000-0002-3695-8347

Uniwersytet Mikołaja Kopernika w Toruniu

\section{Comparaison du fonctionnement des sigles français et polonais dans le discours de la presse}

Skrótowce w polskim i francuskim dyskursie prasowym

Acronyms in the Polish and French press discourse

\section{REMARQUES PRÉLIMINAIRES}

Afin de définir l'objet de notre étude, nous allons rapporter les définitions des sigles dans les deux langues. Pour le français, le travail de référence est celui de L. J. Calvet (1980). Le sigle, effet du processus de la siglaison, y est compris comme «un groupe de mots raccourci en ne conservant que la première lettre de chaque mot» (Calvet, 1980: 7). Il opère en général sur plus d'un mot, contrairement à une abréviation qui est une réduction graphique d'un mot, lue comme un mot entier. «Le sigle est un signe abréviatif formé d'un assemblage de lettres ou groupes de lettres initiales [...] provenant d'un synthème discontinu d'usage fréquent et spécifique, assemblage généralement soumis à des contraintes d'ordre économique et/ou phonique» (Germain and Lapierre, 1988: 63). Le rapport entre le sigle et l'acronyme est défini différemment selon les auteurs; dans ce travail nous comprenons comme acronyme « un groupe de mots raccourcis en conservant le début (le plus souvent la première syllabe) de chaque mot» (Calvet, 1980:7) et considérons l'acronymie comme un sous-ensemble du processus de réduction du signifiant appelé siglaison.

Les définitions des sigles (pl. skrótowce) élaborées par les linguistes polonais sont concordantes. Ainsi H. Jadacka (2002) et J. Młodyński (1981) les définissent comme dérivés des noms d'institutions, entreprises, offices, organisations, associations, etc., formés des lettres ou des syllabes initiales des noms de base. Il est 
à remarquer que dans la définition polonaise, on indique tout de suite comme base de sigles les noms des organismes, ce qui n'est pas le cas pour les travaux français.

La problématique générale adoptée s'intéresse à l'insertion des sigles comme vocables actualisés dans le discours de la presse écrite d'opinion. Pour les sources polonaises, nous proposons une sélection de quotidiens nationaux consultés en ligne: Rzeczpospolita (rp.pl), Gazeta Wyborcza (gazeta.pl) et un hebdomadaire sociétal: Polityka (polityka.pl). Pour les données françaises, nous avons consulté les quotidiens : Le Monde (lemonde.fr), Libération (liberation.fr), Le Figaro (lefigaro. fr) et l'hebdomadaire Le Point (lepoint.fr). L'enquête est limitée à une diachronie de cinq mois de parution, entre les mois de mai et de novembre 2017. Nous nous bornons à une thématique générale d'actualité, celle des rubriques: politique intérieure, société. Notre but est d'observer l'emploi des sigles, non dans les textes de spécialités ou dans un sociolecte précis, mais plutôt dans la langue standard et les textes adressés à un large public. Les sigles d'origine étrangère sont exclus de cette étude focalisée sur les sigles natifs, et surtout sur ceux qui dénomment des réalités socio-culturelles propres aux pays en question.

\section{LES CATÉGORIES DE SIGLES}

Il est banal de dire que la siglaison devient un procédé lexical important et que les sigles se sont implantés dans des domaines d'activités très divers. Leur présence dans la presse générale prouve qu'ils ne font pas toujours partie des termes d'argot de métier mais qu'ils deviennent courants. Intuitivement, nous pouvons dire que la siglaison est un procédé linguistique plus fréquent dans la langue française que polonaise, cette opinion est confirmée par E. Skibińska (2005: 172). Nous tenterons de dégager les principales catégories d'emploi des sigles relevés dans la presse étudiée, afin de mettre en relief d'éventuelles différences entre les presses française et polonaise. Pour ce faire, nous nous inspirons de la classification de Germain et Lapierre (1988), en la modifiant en fonction de notre corpus. Ainsi, nous avons distingué deux grands groupes : le premier constitué d'organismes et de noms de personnes qui relèvent plus des noms propres, et le second, composé des noms communs. À l'intérieur de ce dernier, ont été classés les sigles dénommant: les types d'organisme, les titres, les programmes, les chiffres, les lieux, les objets, et les réalités non classées.

2.1. Organismes et noms de personnes - la plupart des sigles renvoient à des agences, associations, commissions, comités, institutions, organisations administratives, sociétés commerciales et industrielles, universités, partis politiques, syndicats, équipes de sport, etc. Ainsi, nous relevons dans la presse française par 
exemple: CNRS, CCI, CFDT, EDF, ENA, FN, Insee, SNCF, etc.; et dans la presse polonaise, entre autres: RP, SB, UAM, TVP, KRRiT, CBOS, NATO, OFE, ZUS UE, PIS, PO, etc. Il y a dans cette catégorie des sigles venant des toponymes: des noms de pays, d'états ou de régions (fr. DOM-TOM; pl. RP, ZSRR, PRL, etc.). En ce qui concerne les anthroponymes, on observe l'emploi, dans la presse française uniquement, des initiales des noms de personnes célèbres: B. B. (Brigitte Bardot), V.G.E (Valéry Giscard d'Estaing) et DSK (Dominique Strauss-Kahn), etc. Dans la presse polonaise nous ne constatons pas cette tendance, sauf s'il s'agit des célébrités étrangères de la culture populaire, mais non quand on parle des hommes politiques ou d'intellectuels.

2.2. Sigles qui désignent surtout des réalités socioculturelles françaises ou polonaises, répertoriés dans les dictionnaires de langue et considérés comme des noms communs. Ils renvoient à :

2.2.1. des personnes: les membres des organismes (un CRS ou un FFI), des statuts de personnes (un(e) SDF, un(e) OS, un(e) DRH, un PEGC, un(e) PDG). Tous les exemples cités sont français, car dans les textes polonais il est difficile de trouver des sigles désignant des personnes. La seule exception est p.o. qui à l'origine est une abréviation de petniacy obowiązi [faisant fonction], mais qui à l'oral et dans un registre familier est prononcé comme un sigle. Dans d'autres cas, pour désigner des membres d'organismes, la langue polonaise crée des dérivés suffixaux de sigles: akowiec, zomowiec, pisowiec, sokista, ubek, etc.

2.2.2. des types d'organismes, d'associations, de liens. Dans ce groupe, on retrouve entre autres les sigles qui désignent les types d'écoles et de centres de recherche (fr. : ENS, IUT, UFR, CPGE; pl.: LO) ainsi que d'autres types d'établissements : $\mathrm{CHU}$ (fr.) et ZOZ (pl.). Il s'agit aussi bien de noms de réalités propres à la société française (PACS) ou polonaise, que de catégories connues universellement, empruntées à des sciences spécialisées : PME, (pl : MŚP), TPE, ONG, NPI, PMA. Tout de même, pour un grand nombre de ces sigles français, nous ne trouvons pas de sigles polonais correspondants dans la presse polonaise, mais uniquement des termes entiers ou éventuellement, un sigle anglais. À titre d'exemple, pour ONG, nous trouvons en polonais organizacja pozarzadowa ou NGO.

2.2.3. des titres: des noms de brevets, diplômes, certificats (fr. BAFA, BEP, BTS, CAP, CAPA, CAPES, DUT). Nous n'en avons pas relevé d'exemples polonais car dans la langue générale, les diplômes ne sont pas désignés à l'aide des sigles.

2.2.4. des programmes et des systèmes. Cette catégorie comprend entre autres les noms des programmes, des lois et des codes. Nous y incluons aussi, relevés dans les textes français, les droits salariaux (RTT), salaires et allocations (RSA, SMIC), congés, couvertures sociales, assurances (CMU), types de contrats (CDI, CDD). Dans les textes polonais, nous avons trouvé $B H P$ et les noms des assurances: $O C, A C$. 
Ensuite, certains sigles dénomment des soins et des services médicaux (fr.: PMA, IVG, FIV, SAMU; pl. : EKG). Ils dénomment aussi, surtout dans la presse française, des types de cours ou de périodes de la vie scolaire (l'APB, le CE, le CM, le CP, les TP), les matières (les SVT) et les épreuves (les TPE). Dans les textes polonais, on trouve les noms des matières: w.f. ou wuef, WOS ou WDŻ. En français, on lit également des noms de secteurs d'activité et de services (BTP, VTC) et des noms d'impôts (TVA, ISF, IFI).

2.2.5. des chiffres, des nombres, des numéros et des indices, présents dans les deux langues (fr. : le PIB; pl. : PESEL, PKB).

2.2.6. des lieux: des terrains, des zones et des répartitions administratives (fr.: CFA, GR, REP, ZEP, ZAC, ZAD, ZUP, HLM; pl. : JOW).

2.2.7. des objets : (fr.) DAB, GPL, OGM, BD, PV. Il s'agit de noms d'objets de la vie courante, connus dans la société polonaise, mais qui ne sont pas dénommés avec des sigles, sauf les OGM et le GPL pour lesquels on emploie les sigles anglais $G M O$ et $L P G$. En revanche, dans la presse polonaise nous lisons : $A G D, R T V, P C W$ ou $P C V$. Les sigles désignent aussi les véhicules et les moyens de transports (fr. : RER, TER, TGV, ULM, VTT, OVNI; pl.: pks/pekaes).

2.2.8. Dans la catégorie non classés, nous citons les sigles français: JO, JT, NAC, les noms de maladies (IST, MST) et les formules: VO, VF.

Cette classification approximative a pour but de faire apparaitre les différences dans les catégories de fragments de réalité désignés à l'aide des sigles. Les catégories proposées reflètent les tendances sur un échantillon assez restreint de la presse générale, limité de surcroît aux rubriques politique intérieure et société. De plus, dans les médias, la fréquence des occurrences est l'effet de l'actualité, des événements, des débats publics. Tout de même, les différences relevées donnent une certaine idée des divergences existantes dans les deux discours étudiés. Pour les deux langues, nous constatons de nombreux sigles venant des noms d'organismes et fonctionnant comme des noms propres. Toutefois, pour les autres catégories de sigles, nous observons des divergences. Ainsi, dans le discours de la presse française, on emploie des sigles qui dénomment des statuts de personnes (SDF, DRH), des liens entre personnes (PACS), des quartiers (ZEP, REP, ZUP, ZAC, ZAD), des diplômes et niveaux d'études, des cours (CM, TP), des classes d'écoles (CM, CE, CP), des postes, des services médicaux (FIV, PMA, IVG, AMP), des salaires et allocations (SMIC, RSA, RMI), des types de contrat (CDI, CDD), des congés (RTT), des amendes (PV), des secteurs d'activités (BTP) et des objets de la vie quotidienne (CB, VTT, DAB). Par contre, en polonais, si l'on écarte les sigles empruntés à l'anglais et les noms d'organismes, on constate que le recours aux sigles est moins important. Ils dénomment des matières de cours d'école, des impôts ou indices, des soins médicaux et des objets, mais leur éventail est bien plus restreint. 


\section{FONCTION DÉNOMINATIVE LAPIDAIRE}

Grevisse (2006) ou Calvet (1980) reconnaissent la fonction économique des sigles, qui répondent à la loi du moindre effort. Jacqueline Percebois (2001 : 629) qualifie leur rôle de «fonction dénominative lapidaire», commune à tous les sigles et acronymes étant donné qu'ils «facilitent la communication, en synthétisant le concept considéré». Effectivement, dans notre corpus, les sigles largement connus rendent la communication plus rapide non seulement du point de vue de la production du message, mais aussi de sa réception.

3.1. Les sigles des organismes connus des lecteurs sont employés dans les articles de presse sans que l'on cite le nom développé. Ceci concerne des sigles des noms de partis, d'associations, de groupuscules politique ou de syndicats: (fr. : MoDem, LR, LRM, PS, FN, CGT CFDT, FO et CFTC; pl.: PIS, PO, SLD, ONR, KOD), des noms d'institutions internationales (fr.: UE, OTAN, ONU; pl. : UE, ONZ), des sociétés et des institutions nationales, des grandes écoles ou université (fr. : AFP, EDF, ENA, Insee, SNCF; pl. : BOR, ZUS, NFZ, OFE, IPN, GUS, NIK, CBA, RPO, TVP, PAP, KRRiT, KUL, UW). Dans les textes polonais, on emploie aussi des sigles des noms de ministères (MON, MSWiA, MSZ) alors que dans la presse française nous retrouvons plutôt des expressions métonymiques: Matignon, le Quai d'Orsay, etc. Dans les écrits polonais, on retrouve également les sigles des institutions de l'époque précédente: PRL, PZPR, SB, UB, etc. On constate de même l'emploi de sigles qui étaient ignorés du grand public il y a encore quelques mois et qui, sous l'influence de l'actualité, sont très présents dans le discours médiatique et se sont ainsi inscrits dans la mémoire collective (pl.: KRS, TK, SN).

Pour les institutions ou programmes moins connus des lecteurs, la source est accompagnée de son sigle. L'ordre dans lequel ils sont juxtaposés reste changeant, car tantôt le sigle, tantôt le nom entier se retrouve entre parenthèses ou en apposition:

Le Monde a obtenu les chiffres de la répartition des migrants dans les Centres d'accueil et d'orientation (CAO) en France, région par région. (Le Monde, le 26 octobre 2017)

Konrad Tomaszewski, dyrektor generalny Lasów Państwowych (LP), nasze pytania o skalę tegorocznej wycinki nazwał insynuacją i kłamstwem. (Wyborcza, le 22 novembre 2017)

[Konrad Tomaszewski, directeur général des Forêts du domaine public (LP), a qualifié d'insinuation et de mensonge nos questions sur l'ampleur de l'abattage d'arbres de cette année.]

Nous observons aussi, dans les articles français, une tendance à créer des sigles qui apparaissent en même temps que les organismes, les programmes, les 
lois, etc., et qui sont insérés dans le texte malgré leur oralisation difficile, ce qui est le cas du sigle PDMQDC (Plus de maîtres que de classes):

Un dispositif que défend pourtant Stéphane Crochet, de SE-Unsa: «Le PDMQDC apportait le regard croisé de deux enseignants sur les mêmes élèves. En tant que maître, on est chacun dans sa classe avec ses questions, alors que c'était la première fois qu'on partageait ses interrogations et ses choix». (Libération, le 3 septembre 2017)

Ce phénomène est caractéristique de la presse française observée; d'ailleurs, ces sigles sont souvent empruntés à la langue administrative ou technique et constituent une sorte de citation-écho.

3.2. Quant aux sigles communs apparaissant dans la presse française (2.2.), ils sont souvent insérés dans le texte sans leur développement, car ils ont un fonctionnement autonome dans la langue et le discours, en renvoyant directement à un signifié sans que la médiation de la source ne soit nécessaire. Voici, à titre d'exemple, des sigles inclus dans les titres d'articles qui ne sont pas développés non plus dans le texte de l'article:

Hébergement des SDF : situation alarmante dans certains départements français (Le Figaro, le $1^{\text {er }}$ août 2017)

La maire de Paris a adressé une lettre aux 200000 ménages locataires dans une HLM de la capitale. (Le Figaro, le 25 octobre 2017)

VTT alpin: la pratique explose, les accidents aussi (Le Point, le 2 août 2017)

Certains sont si courants qu'ils concurrencent la forme complète à tel point que cette dernière est ignorée. De plus, comme nous verrons plus loin, il arrive que le sigle exprime d'autres sens que ceux de sa source. Il en résulte que le rôle des sigles n'est pas uniquement celui d'abréger la communication.

\section{LE GLISSEMENT DE SENS}

La siglaison, considérée comme procédé de néologie lexicale, crée un signe qui peut avoir une existence autonome : «la forme réduite acquiert le plus souvent une autonomie et une complexité par rapport à la forme dont elle est issue qui relève plus du néologisme que de variante strictement économique» (Ibrahim, 1989: 44). Dans le cas de certains sigles lexicalisés, nous observons une coupure sémantique entre le sigle et sa source (Percebois, 2001 : 641). Ainsi, dans les textes analysés, nous observons des sigles français et polonais qui expriment d'autres sens que leur source. Les noms Compagnie Républicaine de Sécurité ou Państwowa Komunikacja Samochodowa ont pour fonction de nommer des institutions particulières «en 
fixant leur individualité» (Leroy, 2004 : 32). Nous ne pouvons pas dans le cadre de cet article étudier de plus près les critères de l'appartenance des sigles désignant des organismes à la classe des noms propres. Nous nous limiterons à l'observation des sigles CRS et $P K S$ lesquels, au départ, partagent avec leurs sources la même fonction dénominative, en assurant une identification immédiate des institutions considérées comme uniques. Pourtant, nous observons une évolution de leur sens, accompagnée d'une perte de la qualité de «proprium». Dans les exemples qui suivent, on observe un glissement du sens par métonymie ou par permutation, dans la classification des changements de sens d'après Stern (d'après Guiraud, $1975: 48$ ). Ainsi, CRS est le sigle de Compagnie Républicaine de Sécurité, corps spécialisé de la police nationale en France, mais Le Petit Robert note que ce nom désigne un policier membre de ce corps. Voici un exemple d'emploi de ce nom dans ce sens:

L'image d'un $C R S$ atteint par un cocktail Molotov, lors des manifestations du 1er-Mai à Paris, a fait le tour du monde. (Le Monde, le 2 mai 2017)

Le même phénomène se produit dans le cas du sigle polonais $P K S$. Il vient du nom de Państwowa Komunikacja Samochodowa, ancienne entreprise de transport appartenant à l'Etat. Comme il s'agit d'une entreprise qui a été formée en 1945 et qui a existé pendant environ 50 ans, le sigle s'est lexicalisé (Pekaes, pekaes) pour désigner d'abord, par métonymie, un autocar de cette compagnie et ensuite, comme la motivation étymologique s'efface peu à peu, ce mot désigne, par extension ou plutôt par adéquation, selon la typologie de Stern (Guiraud, 1975 : 48), tout autocar qui circule entre les villes:

Wciąż umiem ubrać się tak, by nikt nie zwrócił na mnie uwagi, zmienić się w luja i ruszyć pekaesem w nieznane. Dzikie rozjazdy, zajazdy, parking, Tesco i tyle. No po prostu magiczny świat - mówi „Wyborczej” Michał Witkowski. Właśnie ukazała się jego powieść Wymazane. (Wyborcza, le 21 octobre 2017)

[Je sais toujours m'habiller de façon à ne pas me faire remarquer, me transformer en clodo et partir en 'pekaes' à l'inconnu. Des voies de chemins de fer abandonnées, motels, parking, Tesco, c'est tout. Un monde magique, quoi - dit à «Wyborcza» Michał Witkowski. Son roman Wymazane vient de paraître.]

\section{L'EMPLOI FIGURATIF}

Jacqueline Percebois (2001: 634) distingue au sein des fonctions dénominatives lapidaires, la fonction euphémique. En effet, le rôle euphémisant des sigles en français est étudié par plusieurs linguistes (Bonhomme, Hagège, Horak). La réduction du signifiant le rend moins transparent et entraine sa démotivation: 
une maladie sexuellement transmissible devient MST ou IST, habitation à loyer modéré : HLM, etc. De plus, Horak (2010: 78) observe que les sigles sont parfois «des moyens euphémiques du second degré» parce qu'ils réduisent des expressions étant déjà euphémiques. Ainsi les quartiers pauvres sont dénommés au moyen de termes composés, avec des adjectifs (prioritaires, sensibles) ou avec des expressions prépositionnelles (à urbaniser, de redynamisation) et ensuite au moyen de sigles: QPV (Quartiers prioritaires de la ville), ZUS (Zone urbaine sensible), ZUP (Zone à urbaniser en priorité), ZRU (zones de redynamisation urbaine). Le recours à la siglaison des termes techniques et administratifs donne un effet objectivant et neutralisant quand on aborde les thèmes sensibles de l'actualité politique et sociale. Néanmoins, il serait difficile de dire qu'un sigle est euphémique en soi, indépendamment de son contexte d'emploi et de l'intention du locuteur. Il s'agit plutôt de signes qui sont potentiellement euphémiques en fonction de la situation de communication donnée. Par ailleurs, la reconnaissance d'une unité comme euphémique met en jeu des problèmes axiologiques. En tant que procédé euphémique, le sigle peut connaître un échec, si le récepteur le reconnaît comme tel et le juge inadéquat, et par conséquent s'en distancie ou le démasque (Bonhomme, 2012). De plus, la siglaison est soumise à des critiques, elle est considérée comme une forme de novlangue ou de langue de bois. Dans l'exemple ci-dessous, les dénominations des quartiers pauvres et des programmes de leur réforme (ZFU, ZUS, SRU, ACSEE, PNRU, NPNRU), ainsi que leurs référents, sont l'objet d'une polémique et qualifiées de «barbarismes technocratiques»:

Si l'on s'en tient aux dix dernières années, 43 milliards d'euros auront ainsi été injectés dans les programmes ZFU, ZUS, loi SRU, Halde, plan égalité des chances, ACSEE, commissariat à la diversité, emplois d'avenir, PNRU, NPNRU et autres barbarismes technocratiques. (Le Figaro, le 6 mars 2015)

Dans les textes polonais, nous constatons l'emploi hyperbolique des sigles. Prenons à titre d'exemple le sigle $P G R$, écrit aussi pegeer (Państwowe Gospodarstwo Rolne) qui désigne, d'après le dictionnaire Uniwersalny stownik języka polskiego une grande propriété agricole d'état, sous la République populaire de Pologne et donc caractéristique de l'économie socialiste. Dans l'article cité, le $P G R$ est par extension le synonyme d'une grande propriété agricole, sans qu'elle appartienne à l'État, que ce soit un ancien $P G R$ ou une ferme vieillie ou en ruine. Le texte parle, avec une certaine ironie, des personnes qui ont abandonné les grandes entreprises afin de travailler pour leur propre compte et qui au bout d'un certain temps, y retournent. Le mot $P G R$ est employé ici comme une hyperbole, servant à amplifier la réalité afin d'obtenir un effet comique : 
Nie można odejść zwyczajnie. Trzeba z rozmachem, żeby inni mogli przeczytać: „Były pracownik korpo otworzył wypożyczalnię wielbłądów” - mówi 46-letni Michał Bonarowski [...]. Tekst o nim można zatytułować: „Rzucił korpo i kupił kilkadziesiąt hektarów na Mazurach”. Dom z 1908 roku położony na skarpie. Las. Świeże powietrze. Wizje: on rąbiący drewno (dla przyjemności), on uprawiający topinambur (bo modny), on produkujący sery zagrodowe (przecież lubi sery). Tyle że po sześciu latach powinien powstać tekst nowy: „Sprzedał PGR na Mazurach i z radością wrócił do korpo". (Wyborcza, le 25 septembre 2017)

[On ne peut pas partir ordinairement. Il faut le faire avec éclat pour que les autres puissent lire: "un ancien employé de multinationale a ouvert une location de chameaux - dit Michał Bonarowski de 46 ans [...] Le texte sur lui pourrait être intitulé : «Il a laissé tomber une multinationale et a acheté quelques dizaines d'hectares en Mazurie». Une maison de 1908 sur une colline. La forêt. L'air frais. Des images : lui coupant le bois (par plaisir), cultivant le topinambour (à la mode), fabriquant le fromage artisanal (il aime les fromages). Seulement qu'au bout de six ans, un nouveau texte devrait être écrit: «Il a vendu un $P G R$ en Mazurie et est revenu avec joie à une multinationale.]

\section{CONCLUSION}

L'analyse des sigles de chacune des langues a relevé des différences importantes en ce qui concerne leurs catégories. Dans les deux langues, ils dénomment différents types d'organismes, mais la presse française emploie des sigles qui dénomment des objets de la vie quotidienne, des personnes, des diplômes, etc., qui sont enregistrés dans les dictionnaires de langues, lexicalisés, considérés plutôt comme des noms communs et qui concurrencent les développements qui sont à leur origine. Le procédé de siglaison répond certes au souci d'économie dans le langage, pourtant dans les textes en deux langues nous observons des sigles qui remplissent aussi d'autres fonctions: par glissement de sens, certains véhiculent d'autres sens que les développements, et deviennent des signes linguistiques autonomes, à part entière. D'autre part, le recours aux sigles des termes administratifs pour parler des réalités difficiles, dans les textes non spécialisés est souvent considéré comme procédé euphémique et reste sujet à réussite ou échec discursif en fonction des récepteurs. Il peut aussi provoquer d'autres effets stylistiques, notamment hyperboliques. Cet article ne fait qu'entamer des recherches comparatives sur les sigles dans le discours de presse. La question de la relation entre le sigle et son développement ainsi que l'étude des emplois autres que ceux relevant de la fonction dénominative lapidaire restent à approfondir sur des corpus plus vastes. 


\title{
RÉFÉRENCES BIBLIOGRAPHIQUES
}

Bonhomme, M. (2012). La réception de l'euphémisme: entre réussite et échec interactif. In: A. Horak et al. (eds), Etudes pragmatico-discursives sur l'euphémisme (pp. 73-89). Frankfurt am Main: Peter Lang.

Calvet, J.-L. (1980). Les sigles. Paris: PUF.

Germain, C., Lapierre, A. (1988). Le sigle. Définition, caractéristiques et emploi. Cahiers de lexicologie, 53, 55-74.

Grevisse, M. (2006). Le bon usage: grammaire française. Gembloux: Duculot, De Boeck, Nowela.

Guiraud, P. (1975). La sémantique. Paris: PUF.

Hagège, C. (1996). L'homme de paroles. Contribution linguistique aux sciences humaines. Paris: Fayard.

Horak, A. (2010). L'euphémisme. Entre tradition rhétorique et perspectives nouvelles. Munchen: Lincom Europa.

Ibrahim, A.H. (1989). Productivité par abréviation, réduction ou effacement. Recherches et Applications. Numéro spécial Lexiques, 44-50.

Jadacka, H. (2010). Skrótowce. In: A. Markowski (red.), Wielki słownik poprawnej polszczyzny (pp. 1666-1668). Warszawa: PWN.

Leroy, S. (2004). Le Nom propre en français. Paris: Ophrys.

Młodyński, J. (1981). Skrótowce we współczesnym języku polskim. In: H. Kurkowska (red.), Współczesna polszczyzna (pp. 156-186). Warszawa: PWN.

Percebois, J. (2001). Fonctions et vie des sigles et acronymes en contextes de langues anglaise et française de spécialité. Meta 46(4), 627-645. Https://doi.org/10.7202/003821ar

Skibińska, E. (2005). Skrótowce w polskich przekładach francuskich artykułów prasowych. In: M. Piotrowska (red.), Język trzeciego tysiaclecia III, vol. 2: Konteksty przekładowe (pp.171180). Kraków: Tertium.

\section{RÉSUMÉ}

L'objectif de cet article est de décrire le fonctionnement des sigles en français et en polonais. Bien qu'ils soient présents dans les deux langues, les sigles dans les deux langues ne recouvrent pas les mêmes catégories sémantiques. Dans son étude, l'auteur fait référence aux outils méthodologiques de la néologie lexicale contemporaine. Le procédé de siglaison répond certes au souci d'économie dans le langage, toutefois une étude des emplois des sigles dans le discours de la presse permet de dégager leurs autres fonctions. Ainsi, certains sigles, par glissement de sens, manifestent une autonomie sémantique par rapport à leur source. D'autres se prêtent à des emplois figuratifs, euphémiques ou hyperboliques et sont sujets à des polémiques.

Mots-clés : discours de la presse, figure stylistique, sigles

\begin{abstract}
The purpose of the article is to describe the functioning of acronyms in Polish and French press texts. The acronyms present in both Polish and French press discourse are generally used differently as disparate semantic categories. Even though the organizational acronyms dominate the discourses, in the French texts other categories are also visible. The theories and methods of word-formation of both languages were used in the work. Using acronyms is obviously symptomatic for linguistic
\end{abstract}


tendencies to economize on means of expression, however, a study of their discursive usage allows to differentiate lexical acronyms with a meaning different than that of the full name. Moreover, acronyms also function as stylistic devices equivalent to euphemism or hyperbole.

Keywords: acronyms, figure of speech, press discourse

\begin{abstract}
ABSTRAKT
Celem artykułu jest ukazanie, że zarówno francuski, jak i polski dyskurs prasowy charakteryzują się użyciem skrótowców, jednak te ostatnie różnią się pod względem kategorii semantycznych. Chociaż w obu dyskursach dominują skrótowce od nazw organizmów, to w tekstach francuskich są obecne także akronimy innych kategorii. W pracy zostały wykorzystane teorie i metody słowotwórstwa obu języków. Stosowanie form abrewiacyjnych jest przejawem tendencji języka do ekonomiczności, ale obserwacja ich funkcjonowania w tekstach prasowych pozwala wyodrębnić także inne efekty stylistyczne i pragmatyczne. Można wyróżnić skrótowce zleksykalizowane o znaczeniu innym od znaczenia ich rozwinięcia. Niektóre z nich służą za eufemizmy, inne wpływają na hiperbolizację wypowiedzi lub stają się przedmiotem polemiki.
\end{abstract}

Słowa kluczowe: dyskurs prasowy, figura stylistyczna, skrótowce 
